\title{
Pandora: Description of a Painting Database for Art Movement Recognition with Baselines and Perspectives
}

Corneliu Florea

corneliu.florea@upb.ro;

Ră̌zvan Condorovici

1condorovici@imag.pub.ro;

Constantin Vertan

constantin.vertan@upb.ro;

Raluca Boia

rboia@imag.pub.ro;

Laura Florea

viaura.florea@upb.ro;

Ruxandra Vrânceanu

yranceanu@imag.pub.ro;
Image Processing and Analysis

Laboratory

University "Politehnica" of Bucharest

Bucharest, Romania

\begin{abstract}
To facilitate computer analysis of visual art, in the form of paintings, we introduce Pandora (Paintings Dataset for Recognizing the Art movement) database, a collection of digitized paintings labelled with respect to the artistic movement. Noting that the set of databases available as benchmarks for evaluation is highly reduced and most existing ones are limited in variability and number of images, we propose a novel large scale dataset of digital paintings. The database consists of more than 7700 images from 12 art movements. Each genre is illustrated by a number of images varying from 250 to nearly 1000. We investigate how local and global features and classification systems are able to recognize the art movement. Our experimental results suggest that accurate recognition is achievable by a combination of various categories.
\end{abstract}

\section{Introduction}

The remarkable expansion of the digital data during the last period favored a much easier access to works of art for the general public. Great efforts were put lately into creating automatic image processing solutions that facilitate a better understanding of art [ $[$ ]. These solutions may aim at obtaining high-quality and high-fidelity digital versions of paintings $[\square]$ or may address various aspects such as: image diagnostics, virtual restoration, color rejuvenation etc. as discussed in the review of Stork et al. []. Another more appropriate to the ultimate goal of computers is the context recognition. One of the broadest possible implementation of context recognition is the automatic art movement identification. 
According to Artyfactory [四, art movements are "collective titles that are given to artworks which share the same artistic ideals, style, technical approach or timeframe". While some works are clearly set into a single art movement, others are in the transition period, as painters loved to experiment new ideas, leading to creation of a new movement. Also while the actual characteristics place a work in some art movement, its author, for personal reasons, refused to be categorized in such a way, giving birth to disputes.

In this paper, we look into the problem of computational categorization of digitized paintings into artistic genres (or art movements). In contrast to other directions of image classification, such as scene or object recognition, where large databases and evaluation protocols do exist, such an aspect is less emphasized for digitized paintings. Typically, the evaluation of a new method is carried on a small database with few paintings belonging to few genres. Given the latest advances of machine learning, two aspects should be noted: (1) deep networks with the many parameters easily overfit on small databases and (2) to have progress, we need larger databases.

In this paper we start by reviewing painting collections introduced in prior art and we follow by describing the proposed database. Next, to form a baseline, we continue by reporting the performance of various popular image descriptors and machine learning systems on the introduced database. The paper ends with discussions and conclusions.

\section{Related work}

In the last period multiple solutions issued automatic analysis of visual art and especially paintings using computer vision techniques. However, most of the research is based on medium-to-small databases. A summary of such methods is presented in table 1. One may easily note the size of the databases (and implicitly the number of art movements investigated) increased with time, while the reported performance decreased until it stabilized in the range of 50-70\% for correct art movement recognition. Some of the most representative databases used for art movement identification are:

- Artistic genre dataset [ $\square]$. Images, gathered from Web Museum-Paris, were set in the following art movements: Classicism, Cubism, Impressionism, Surrealism, Expressionism.

- Artistic genre dataset [ $[\mathbb{0}]$. Images from various Internet sources were categorized into 5 genres : Abstract, Impressionism, Cubism, Pop Art and Realism.

- Painting genre dataset $[\square]$ : Images collected from the Internet were grouped into: Abstract expressionist, Baroque, Cubist, Graffiti, Impressionist and Renaissance.

- Artistic style dataset $[\square]$ : Paintings from 9 painters were grouped intro three art movements: Impressionism, Abstract expressionism and Surrealism.

- Artistic genre dataset [ $[$ ] with images collected from Artchive fine-art dataset and grouped into: fine-art genres: Renaissance, Baroque, Impressionism, Cubism, Abstract, Expressionism and Pop art.

- Paintings-91 dataset [ $\square]$ with images collected from the Internet. While the database is larger than the previous ones, only paintings corresponding to painters that have the majority of works into one art movement got a genre label. It resulted in a smaller 
Table 1: Art movement recognition solutions with the size of used databases. The database size refers only to the database used for art movement recognition, as in some cases larger databases have been implied for other purposes. The value for recognition rate (RR) is the one reported by the respective work while the "test ratio" is the percentage used for testing from the overall database (CV-stands for cross validation). We kindly ask the reader to retrieve all the details from the respective work.

\begin{tabular}{|c|c|c|c|c|}
\hline Method & $\begin{array}{l}\text { Move- } \\
\text { ments }\end{array}$ & $\begin{array}{l}\text { Db. } \\
\text { size }\end{array}$ & RR. & Test ratio \\
\hline Gunsel et al. [四] & 3 & 107 & $91.66 \%$ & $53.5 \%$ \\
\hline Zujovic et al. [四] & 5 & 353 & $68.3 \%$ & $10 \% \mathrm{CV}$ \\
\hline Siddiquie et al. [四] & 6 & 498 & $82.4 \%$ & $20 \% \mathrm{CV}$ \\
\hline Shamir et al. [ㅁ] & 3 & 517 & $91 \%$ & $29.8 \%$ \\
\hline Arora\&ElGammal[G] & 7 & 490 & $65.4 \%$ & $20 \% \mathrm{CV}$ \\
\hline Khan et al. [ष] & 13 & 2338 & $62.2 \%$ & $46.53 \% \mathrm{CV}$ \\
\hline Condorovici et al.[] & 8 & 4119 & $72.24 \%$ & $10 \% \mathrm{CV}$ \\
\hline Agarwal et al. [घ] & 10 & 3000 & $62.37 \%$ & $10 \% \mathrm{CV}$ \\
\hline$\overline{\text { Proposed }}$ & 12 & $\overline{7724}$ & $54.7 \%$ & $25 \% C V$ \\
\hline
\end{tabular}

database illustrating Abstract expressionism, Baroque, Constructivism, Cubism, Impressionism, Neo-classical, Pop art, Post-impressionism, Realism, Renaissance, Romanticism, Surrealism and Symbolism. Probably this is the most structured database previously proposed.

- Artistic genre dataset [ $[$ ] is the basis of the proposed database. We increased that dataset by adding more images to illustrate the existing art movements and added 4 new ones.

- Artistic genre dataset [■] contains images collected from WikiArt and grouped into: Abstract-expressionism, Baroque, Cubism, Impressionism, Expressionism, Pop Art, Rococo, Realism, Renaissance and Surrealism.

Concluding, many of the databases previously used, are small and contain non-standard evaluation protocols allowing overfitting. Thus, a larger scale database with fixed evaluation protocol should be beneficial for further development on the topic.

\section{Pandora database}

Our main contribution is the creation of a new and extensive dataset of art images ${ }^{1}$. While we follow the Paintings-91 database [ $\square$ ] , our dataset is significantly larger, it was build around art movements and not painters and we tried to span wider time periods from antiquity to current periods. The later property should help the automatic study of style evolution, of thematic evolution and cross-time relationship identifications.

The Pandora (Paintings Dataset for Recognizing the Art movement) dataset consists of 7724 images from 12 movements: old Greek pottery, iconoclasm, high renaissance, baroque,

\footnotetext{
${ }^{1}$ The up-to-date database with pre-computed features data reported here is available at http://imag.pub. ro/pandora/pandora_download.html
} 
Table 2: The structure of the Pandora database.

\begin{tabular}{|c|c|c|}
\hline Art movement & No. of paintings & Historical period \\
\hline Old Greek pottery & 350 & Antiquity \\
\hline Iconoclasm & 665 & Middle age \\
\hline High renaissance & 812 & $1490-1527$ \\
\hline Baroque & 960 & $1590-1725$ \\
\hline Rococo & 844 & $1650-1850$ \\
\hline Romanticism & 874 & $1770-1850$ \\
\hline Impressionism & 984 & $1860-1925$ \\
\hline Realism & 307 & $1848-$ present \\
\hline Cubism & 920 & $1900-$ present \\
\hline Abstract-expressionism & 340 & $1920-$ present \\
\hline Fauvism & 426 & $1900-1950$ \\
\hline Surrealism & 242 & $1900-$ present \\
\hline
\end{tabular}

rococo, romanticism, impressionism, realism, cubism, fauvism, abstract-expressionism and surrealism. The precise database structure is shown in table 2 and some examples representative for the art movements are in figure 1. We kindly ask the reader to note some of difficulties in distinguishing between genres: the main difference between Abstract and Fauvism is the less natural order in the structure of the Abstract works, while the Fauvism tends "to use color to express joy". Baroque has a darker tone with respect to Romanticism while the later depicts "exotism or extraordinary things" . The difference between Realism and Surrealism is that the later illustrate "irrational juxtaposition of images" [四 (e.g. such as wings attached to the girl). Yet thinking in computer terms, to detect the irrational of joy in an image is extremely hard. Thus we consider that to achieve such goals, one needs, first, an appropriate database of considerable size and variability.

\section{Art movement recognition performance}

\subsection{Training and testing}

To separate the database training and testing parts, a 4-fold cross validation scheme was implemented. The division into 4 folds exists at the level of each art movement, thus each image being uniquely allocated into a fold. The same division was used for all further tests and it is part of the database.

\subsection{Features and classifiers}

As "there is no fixed rule that determines what constitutes an art movement" and "the artists associated with one movement may adhere to strict guiding principles, whereas those who belong to another may have little in common" [四, there cannot be a single set of descriptors that are able to separate any two art movements.

Following the observations from prior works [], [凹] , multiple categories of feature descriptors should be used. For instance, to differentiate between impressionism and previous styles, one of the main difference is the brush stroke, thus texture. Old Pottery and Orthodox 


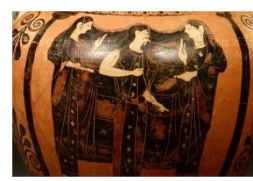

Old Greek Pottery



Rococo

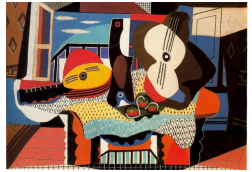

Cubism

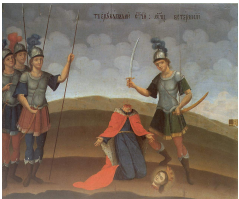

Orthodox Iconoclasm

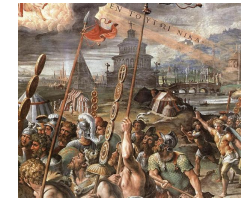

High Renaissance

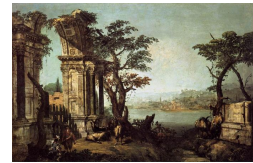

Baroque

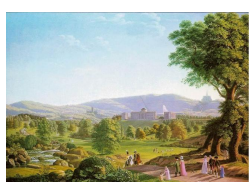

Romanticism

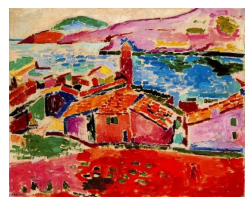

Fauvism
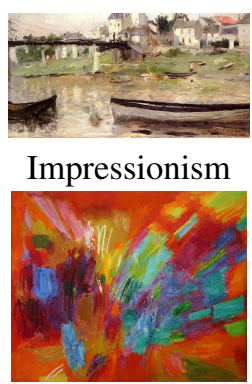

Abstract

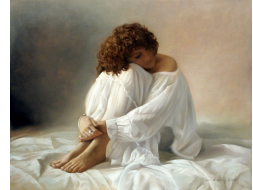

Realism

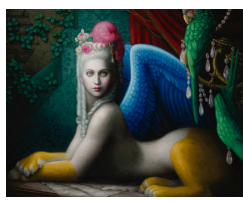

Surrealism

Figure 1: The 12 art movements illustrated in the proposed database.

Iconoclasm are older and use a limited color palette. Also, one needs to understand the content of the painting to distinguish between realism and surrealism (for instance); thus, global composition descriptor should be used.

To provide a baseline for further evaluation, we have tested various combinations of popular feature extractors and classification algorithms.

The texture feature extractors used are :

- Histogram of oriented gradients (HOG) [ $[$ ] which computes the oriented gradient in each pixel and accumulates the weight of each orientation into a histogram. It has

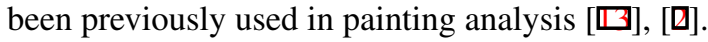

- Pyramidal HOG (pHOG) the above mentioned HOG is implemented on 4 levels of a Gaussian pyramid.

- Color HOG - the above mentioned HOG descriptor applied on each color plane of the RGB color space.

- Local Binary Pattern (LPB) [प] is a histogram of quantized binary patterns pooled in a local image neighborhood of $3 \times 3$ and restrained to a total of 58 quantized non-

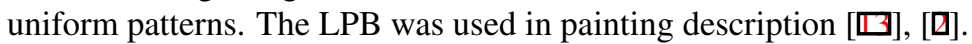

- Pyramidal LBP (pLBP) - the above mentioned descriptor computed over 4 levels of a Gaussian pyramid.

- Local Invariant Order Pattern [] - assume the order after sorting in the increasing intensity local samples.

For HOG, LBP and LIOP we have relied on the implementation from the VLFeat library [四]. 
- Edge Histogram Descriptor (EHD) is part of the MPEG-7 standard. It accounts for the distribution of four basic gradient orientations within regular image parts. The implementation is based on BilVideo-7 library [四].

- The spatial envelope, GIST [ $\square$ ] describes the spatial character or shape of the painting and was previously used for painting categorization [ $[\square]$.

The color descriptors tested are:

- Discriminative Color Names (DCN) [四] - represents the dominant color retrieved through an information oriented approach. Here, we have used author provided code. The baseline form (Color Name) was successfully used to determine the style and the painter [四].

- Color Structure Descriptor (CSD) [四], which is based on color structure histogram, a generalization of the color histogram. The CSD accounts for some spatial coherence in the gross distribution of quantized colors within the image and it has been shown that is able to differentiate between various art movements [] . We computed a 64 long CSD vector using the BilVideo-7 library [0].

Machine learning classification systems tested are:

- Support Vector Machine. We have relied for its implementation on the Lib-SVM [छ]. We used on the radial basis function c-SVM and followed, for each case, the optimization (i.e. exhaustive search in (cost,gamma) space) recommended by the LibSVM creators.

- Random forest [ब]. We have used 100 trees and unlimited depth. At each node we randomly look for a split in $N_{1}=\sqrt{N}$ dimensions where $N$ is the input feature dimension.

Let us note that before the development of the deep networks the random forests and support vector machines have been found to be the most robust families of classifiers [四]. Also, for small and diverse databases SVM and RF out-compete deep networks.

- k-Nearest neighbor (kNN). We have implemented 1-NN, 3-NN and 7-NN based on Euclidean distance. While we report the results in terms of correct recognition rate, the nearest neighbor results will give an indication about the retrieval performance as it may be translated in terms of precision-recall.

Furthermore we have tested several systems that were previously used for art movement recognition. Inspired from previous work [ [] , we have run the Bag of Words (BoW) over SIFT keypoint detector with a vocabulary of 500. We have also tested a combination of color description, texture analysis based on Gabor filters and scene composition based on Gestalt frameworks [0].

Additionally, while the database is small for such a purpose and thus not really suited for deep learning, to have an indication of baseline performance, we have trained and evaluated a version of Deep Convolutional Neural Network (CNN). Our implementation is based on the MatConvNet [四] library and LeNet architecture [四]. 
Table 3: Recognition rates when various combinations of features and classifiers are used on the Pandora database. We marked with bold the best performance.

\begin{tabular}{|c|c|c|c|c|c|}
\hline Feat. / Class. & Random Forest & SVM & 1-NN & 3-NN & 7-NN \\
\hline HOG & 0.266 & 0.248 & 0.200 & 0.214 & 0.233 \\
\hline pHOG & 0.342 & 0.364 & 0.262 & 0.266 & 0.267 \\
\hline colorHOG & 0.268 & 0.277 & 0.213 & 0.221 & 0.236 \\
\hline LBP & 0.386 & 0.395 & 0.303 & 0.298 & 0.320 \\
\hline pLBP & 0.459 & 0.525 & 0.368 & 0.362 & 0.377 \\
\hline LIOP & 0.344 & 0.362 & 0.246 & 0.252 & 0.260 \\
\hline EHD & 0.319 & 0.287 & 0.270 & 0.267 & 0.286 \\
\hline \hline GIST & 0.379 & 0.337 & 0.297 & 0.280 & 0.282 \\
\hline \hline DCN & 0.298 & 0.264 & 0.192 & 0.201 & 0.215 \\
\hline CSD & 0.435 & 0.489 & 0.337 & 0.3357 & 0.363 \\
\hline \hline pLBP + DCN & 0.488 & 0.521 & 0.278 & 0.282 & 0.297 \\
\hline \hline pLBP + CSD & 0.540 & $\mathbf{0 . 5 4 7}$ & 0.377 & 0.282 & 0.297 \\
\hline
\end{tabular}

\subsection{Results}

We report first the results achieved when various combinations of features and classifiers are used (to be followed in table 3). We also report, in tables 4, 5, the confusion matrices for the best combination in each category: $\mathrm{pLPB}+\mathrm{SVM}, \mathrm{GIST}+\mathrm{RF}, \mathrm{CSD}+\mathrm{SVM}$ and respectively pLBP+CSD+SVM.

Secondly we report comparatively the best performance of aggregated systems in table 6. We note that for this particular database, the best performance is achieved by a standard combination of features (pyramidal LBP + Color Structure Descriptor) with a Support Vector Machine.

While one may find disappointing the performance of various established systems, this is perfectly explainable. For the Bag of Words there is too much variability between keypoints to find a common ground; instead of the baseline version tested here, one should opt for much larger vocabularies with accurate compression to keep memory requirements low. Regarding the performance of the DeepCNN, the reported value should be perceived as a lower boundary, as the database is too small for directly training nets with tens of thousands of variables, since no data augmentation was implemented and the images being resized at $32 \times 32$ lost some of the defining characteristics.

\section{Discussion and conclusions}

The best achieved performance was by a combination of pyramidal LBP and Color Structure Descriptor. One may expect the addition of GIST to further increase the performance, but this does not happen, probably due to the curse of dimensionality (the features dimension reaching 800); in such a case a feature selection method should be used, but we consider it outside the scope of the current paper.

The next important observation is that different descriptors do a good job separating some currents and not so good on identifying others. For instance, the CSD separates excellently the Orthodox Iconoclasm which has a unique color palette (due to degradation in time and reduced colors available at creation), but it is not able to separate Fauvism from 
Table 4: Confusion Matrices for the best performers on each category. The art movement used acronyms are: old Greek pottery-Gre, iconoclasm-Ico, high renaissance-Ren, baroque-Bar, rococo-Roc, romanticism-Rom, impressionism-Impr, realism-Real, cubismcub, fauvism-Fauv, abstract-expressionism-Abs and surrealism-Sur.

\begin{tabular}{|c|c|c|c|c|c|c|c|c|c|c|c|c|c|}
\hline & Gre & Ren & Icon & Roc & Rom & Bar & Impr & Cub & Abs & Fauv & Real & Sur \\
\hline Gre & $\mathbf{2 0 0}$ & 20 & 28 & 2 & 3 & 5 & 35 & 51 & 3 & 1 & 0 & 2 \\
\hline Ren & 5 & $\mathbf{3 6 0}$ & 24 & 54 & 47 & 153 & 95 & 70 & 1 & 2 & 0 & 1 \\
\hline Icon & 7 & 21 & $\mathbf{5 5 9}$ & 7 & 1 & 15 & 23 & 30 & 2 & 0 & 0 & 0 \\
\hline Roc & 4 & 90 & 21 & $\mathbf{2 7 2}$ & 129 & 214 & 73 & 38 & 1 & 0 & 0 & 2 \\
\hline Rom & 9 & 106 & 16 & 165 & $\mathbf{2 1 9}$ & 183 & 107 & 55 & 2 & 5 & 3 & 4 \\
\hline Bar & 4 & 173 & 21 & 186 & 121 & $\mathbf{2 9 2}$ & 87 & 65 & 2 & 3 & 0 & 6 \\
\hline Impr & 10 & 97 & 47 & 66 & 106 & 95 & $\mathbf{4 2 9}$ & 109 & 8 & 6 & 2 & 9 \\
\hline Cub & 14 & 93 & 65 & 42 & 55 & 76 & 167 & $\mathbf{3 6 1}$ & 12 & 12 & 2 & 21 \\
\hline Abs & 11 & 43 & 43 & 5 & 18 & 11 & 66 & 67 & $\mathbf{4 6}$ & 2 & 0 & 28 \\
\hline Fauv & 7 & 53 & 20 & 15 & 35 & 43 & 118 & 89 & 6 & $\mathbf{1 8}$ & 1 & 21 \\
\hline Real & 2 & 10 & 16 & 22 & 25 & 17 & 39 & 16 & 1 & 3 & $\mathbf{1 3 5}$ & 21 \\
\hline Surr & 2 & 13 & 16 & 7 & 16 & 7 & 43 & 37 & 14 & 7 & 2 & $\mathbf{7 8}$ \\
\hline
\end{tabular}

GIST + RF

\begin{tabular}{|c|c|c|c|c|c|c|c|c|c|c|c|c|}
\hline & Gre & Ren & Icon & Roc & Rom & Bar & Impr & Cub & Abs & Fauv & Real & Sur \\
\hline Gre & $\mathbf{3 3 9}$ & 4 & 1 & 1 & 0 & 3 & 0 & 1 & 0 & 0 & 1 & 0 \\
\hline Ren & 0 & $\mathbf{4 0 4}$ & 16 & 49 & 66 & 219 & 24 & 31 & 0 & 2 & 1 & 0 \\
\hline Icon & 0 & 8 & $\mathbf{5 7 6}$ & 3 & 0 & 6 & 30 & 31 & 0 & 11 & 0 & 0 \\
\hline Roc & 0 & 118 & 2 & $\mathbf{2 3 0}$ & 153 & 262 & 51 & 18 & 0 & 1 & 8 & 1 \\
\hline Rom & 0 & 91 & 4 & 113 & $\mathbf{3 2 2}$ & 228 & 74 & 31 & 0 & 1 & 10 & 0 \\
\hline Bar & 0 & 178 & 8 & 152 & 150 & $\mathbf{4 0 5}$ & 42 & 18 & 0 & 1 & 5 & 1 \\
\hline Impr & 0 & 24 & 58 & 33 & 35 & 66 & $\mathbf{5 8 4}$ & 133 & 13 & 29 & 9 & 0 \\
\hline Cub & 2 & 43 & 42 & 38 & 21 & 32 & 168 & $\mathbf{5 0 8}$ & 16 & 30 & 5 & 15 \\
\hline Abs & 0 & 3 & 11 & 1 & 7 & 2 & 55 & 83 & $\mathbf{1 2 5}$ & 45 & 0 & 8 \\
\hline Fauv & 0 & 9 & 15 & 8 & 8 & 11 & 104 & 85 & 34 & $\mathbf{1 4 5}$ & 3 & 4 \\
\hline Real & 1 & 18 & 14 & 30 & 29 & 42 & 69 & 38 & 4 & 7 & $\mathbf{5 0}$ & 5 \\
\hline Surr & 0 & 3 & 5 & 8 & 16 & 5 & 44 & 65 & 15 & 19 & 5 & $\mathbf{5 7}$ \\
\hline
\end{tabular}

CSD + SVM

Impressionism as both use the same colors but distributed differently. The Surrealism is hard to separate by everything else except GIST as it is the only tested feature able to describe the scene composition. Yet the GIST is not able to distinguish the Fauvism from Impressionism as local texture makes the difference. In contrast, the pLBP confusion between Fauvism and Impressionism is much reduced.

Overall, the confusion between Abstract and Cubism is large. As Cubism is defined by the extraordinary apparition of straight lines, to address it, one should try to introduce features appropriate to describe rectilinear objects.

Concluding we propose a new painting database annotated with art movements labels and divided in 4 folds to prepare it for rigorous evaluation. The database is significantly larger than the ones previously used. We have tested a multitude of popular features and classifiers and we have identified the weak and strong points of each of them. We also suggest some directions for future research that we anticipate to be beneficial for progress in the field. 


\section{Acknowledgment}

This work is supported by a grant of the Romanian National Authority for Scientific Research and Innovation, CNCS Ú UEFISCDI, number PN-II-RU-TE-2014-4-0733

\section{References}

[1] What is an art movement? http://www.artyfactory.com/art_ appreciation/art_movements/art_movements.htm, Retrieved February 2016.

[2] S. Agarwal, H. Karnick, N. Pant, and U. Patel. Genre and style based painting classification. In Proc. of WACV, pages 588-594, 2015.

[3] R. S. Arora, , and Ahmed Elgammal. Towards automated classification of fine-art painting style: a comparative study. In Proc. of ICPR, pages 3541-3544, 2012.

[4] Muhammet Baştan, Hayati Çam, Uğur Güdükbay, and Özgür Ulusoy. BilVideo-7: An MPEG-7-Compatible Video Indexing and Retrieval System. IEEE MultiMedia, 17(3): 62-73, 2009.

[5] Leo Breiman. Random forests. Machine learning, 45(1):5-32, 2001.

[6] Chih-Chung Chang and Chih-Jen Lin. Libsvm: A library for support vector machines. ACM Trans. Intell. Syst. Technol., 2(3), May 2011.

[7] Razvan George Condorovici, Corneliu Florea, and Constantin Vertan. Automatically classifying paintings with perceptual inspired descriptors. J. Vis. Commun. Image. Represent., 26:222 - 230, 2015.

[8] B. Cornelis, A. Dooms, J. Cornelis, F. Leen, and P. Schelkens. Digital painting analysis, at the cross section of engineering, mathematics and culture. In Proc. of EUSIPCO, pages 1254-1259, 2011.

[9] N. Dalal and B. Triggs. Histograms of oriented gradients for human detection. In Proc. of CVPR, pages 886-893, 2005.

[10] Manuel Fernández-Delgado, Eva Cernadas, Senén Barro, and Dinani Amorim. Do we need hundreds of classifiers to solve real world classification problems? JMLR, 15(1): 3133-3181, 2014.

[11] B. Gunsel, S. Sariel, and O. Icoglu. Content-based access to art paintings. In Proc. of ICIP, pages 558-561, 2005.

[12] Yin-Fu Huang and Chang-Tai Wang. Classification of painting genres based on feature selection. In Proc. of Multimedia and Ubiquitous Engineering, LNEE, volume 308, pages 159-164, 2014.

[13] Fahad Shahbaz Khan, Shida Beigpour, Joost van de Weijer, and Michael Felsberg. Painting-91: a large scale database for computational painting categorization. Mach. Vis. App., 25(6):1385-1397, 2014. 
[14] R. Khan, J. van de Weijer, F. Shahbaz Khan, D. Muselet, C. Ducottet, and C. Barat. Discriminative color descriptors. In Proc. of CVPR, pages 2866-2873, 2013.

[15] Y. LeCun, L. Bottou, Y. Bengio, and P. Haffner. Gradient-based learning applied to document recognition. Proceedings of the IEEE, 86(11):2278-2324, 1998.

[16] B. S. Manjunath, J. R. Ohm, V. V. Vasudevan, and A. Yamada. Color and texture descriptors. IEEE Trans. Cir. and Sys. for Video Technol., 11(6):703-715, 2001.

[17] K. Martinez, J. Cupitt, D. Saunders, and R. Pillay. Ten years of art imaging research. Proceedings of the IEEE, 90(1):28-41, 2002.

[18] Timo Ojala, Matti Pietikäinen, and Topi Mäenpää. Multiresolution gray-scale and rotation invariant texture classification with local binary patterns. IEEE Trans. Pattern Anal. Mach. Intell., 24(7):971-987, July 2002.

[19] Aude Oliva and Antonio Torralba. Modeling the shape of the scene: A holistic representation of the spatial envelope. IJCV, 42(3):145-175, 2001.

[20] Lior Shamir, Tomasz Macura, Nikita Orlov, D. Mark Eckley, and Ilya G. Goldberg. Impressionism, expressionism, surrealism: Automated recognition of painters and schools of art. ACM Trans Appl Percept, 7(2):1-17, 2010.

[21] B. Siddiquie, S.N. Vitaladevuni, and L.S. Davis. Combining multiple kernels for efficient image classification. In Proc. of WACV, pages 1-8, 2009.

[22] D. Stork. Computer vision and computer graphics analysis of paintings and drawings: An introduction to the literature. In Proc. of CAIP, pages 9-24, 2009.

[23] Andrea Vedaldi and Brian Fulkerson. Vlfeat: An open and portable library of computer vision algorithms. In Proc. of ACM MM, pages 1469-1472, 2010.

[24] Andrea Vedaldi and Karel Lenc. Matconvnet: Convolutional neural networks for matlab. In Proc. of ACM MM, pages 689-692, 2015.

[25] Zhenhua Wang, Bin Fan, and Fuchao Wu. Local intensity order pattern for feature description. In Proc. of ICCV, pages 603-610, 2011.

[26] J. Zujovic, L. Gandy, S. Friedman, B. Pardo, and T.N. Pappas. Classifying paintings by artistic genre: An analysis of features \& classifiers. In Proc. of IEEE MMSP, pages $1-5,2009$. 
Table 5: Confusion Matrices for the best performers on each category. The art movement used acronyms are: old Greek pottery-Gre, iconoclasm-Ico, high renaissance-Ren, baroque-Bar, rococo-Roc, romanticism-Rom, impressionism-Impr, realism-Real, cubism$c u b$, fauvism-Fauv, abstract-expressionism-Abs and surrealism-Sur.

\begin{tabular}{|c|c|c|c|c|c|c|c|c|c|c|c|c|}
\hline & Gre & Ren & Icon & Roc & Rom & Bar & Impr & Cub & Abs & Fauv & Real & Sur \\
\hline Gre & $\mathbf{2 7 1}$ & 4 & 2 & 1 & 5 & 1 & 11 & 27 & 5 & 18 & 2 & 3 \\
\hline Ren & 4 & $\mathbf{4 4 4}$ & 1 & 40 & 60 & 166 & 36 & 58 & 1 & 0 & 1 & 1 \\
\hline Icon & 42 & 1 & $\mathbf{5 9 8}$ & 1 & 0 & 3 & 3 & 10 & 3 & 4 & 0 & 0 \\
\hline Roco & 0 & 48 & 3 & $\mathbf{3 2 4}$ & 136 & 232 & 63 & 36 & 0 & 0 & 1 & 1 \\
\hline Rom & 3 & 94 & 4 & 139 & $\mathbf{3 3 9}$ & 182 & 80 & 27 & 1 & 3 & 0 & 2 \\
\hline Bar & 1 & 126 & 3 & 165 & 136 & $\mathbf{4 4 1}$ & 47 & 37 & 0 & 2 & 0 & 2 \\
\hline Impr & 9 & 29 & 10 & 49 & 92 & 43 & $\mathbf{6 1 8}$ & 112 & 6 & 14 & 1 & 1 \\
\hline Cub & 63 & 29 & 23 & 17 & 29 & 28 & 128 & $\mathbf{5 7 0}$ & 8 & 17 & 0 & 8 \\
\hline Abs & 20 & 11 & 21 & 7 & 22 & 17 & 56 & 91 & $\mathbf{5 9}$ & 23 & 2 & 11 \\
\hline Fauv & 33 & 5 & 5 & 1 & 18 & 9 & 111 & 58 & 11 & $\mathbf{1 6 3}$ & 2 & 10 \\
\hline Real & 15 & 11 & 4 & 7 & 17 & 17 & 33 & 23 & 13 & 18 & $\mathbf{1 4 1}$ & 8 \\
\hline Surr & 18 & 13 & 10 & 4 & 23 & 19 & 17 & 40 & 13 & 13 & 4 & $\mathbf{6 8}$ \\
\hline
\end{tabular}

\begin{tabular}{|c|c|c|c|c|c|c|c|c|c|c|c|c|c|}
\hline & Gre & Ren & Icon & Roc & Rom & Bar & Impr & Cub & Abs & Fauv & Real & Sur \\
\hline Gre & $\mathbf{3 0 7}$ & 0 & 0 & 0 & 0 & 0 & 2 & 40 & 0 & 0 & 1 & 0 \\
\hline Ren & 0 & $\mathbf{4 7 3}$ & 2 & 49 & 63 & 159 & 24 & 41 & 0 & 1 & 0 & 0 \\
\hline Icon & 0 & 1 & $\mathbf{6 1 6}$ & 1 & 1 & 2 & 10 & 31 & 1 & 2 & 0 & 0 \\
\hline Roc & 0 & 83 & 1 & $\mathbf{3 0 1}$ & 163 & 214 & 59 & 23 & 0 & 0 & 0 & 0 \\
\hline Rom & 0 & 77 & 0 & 104 & $\mathbf{3 7 2}$ & 184 & 74 & 63 & 0 & 0 & 0 & 0 \\
\hline Bar & 0 & 132 & 0 & 169 & 148 & $\mathbf{4 3 7}$ & 50 & 24 & 0 & 0 & 0 & 0 \\
\hline Impr & 0 & 17 & 7 & 31 & 43 & 36 & $\mathbf{6 9 6}$ & 143 & 0 & 10 & 1 & 0 \\
\hline Cub & 2 & 21 & 13 & 11 & 21 & 17 & 119 & $\mathbf{7 0 6}$ & 1 & 9 & 0 & 0 \\
\hline Abs & 0 & 0 & 4 & 1 & 6 & 1 & 53 & 200 & $\mathbf{6 2}$ & 13 & 0 & 0 \\
\hline Fauv & 0 & 2 & 2 & 2 & 6 & 1 & 126 & 190 & 3 & $\mathbf{9 3}$ & 1 & 0 \\
\hline Real & 0 & 3 & 0 & 9 & 13 & 11 & 56 & 79 & 0 & 4 & $\mathbf{1 3 1}$ & 1 \\
\hline Surr & 0 & 1 & 3 & 4 & 7 & 2 & 33 & 159 & 4 & 6 & 0 & $\mathbf{2 3}$ \\
\hline
\end{tabular}

$$
\mathrm{pLBP}+\mathrm{CSD}+\mathrm{SVM}
$$

Table 6: Recognition rates when various systems are used.

\begin{tabular}{|c|c|}
\hline System & Performance \\
\hline pLBP + CSD +SVM & $\mathbf{0 . 5 4 7}$ \\
\hline BoW & 0.352 \\
\hline Condorovici et al. [ [ ] & 0.379 \\
\hline Deep CNN & 0.486 \\
\hline
\end{tabular}

